PROCEEDINGS OF THE

AMERICAN MATHEMATICAL SOCIETY

Volume 138, Number 5, May 2010, Pages 1843-1852

S 0002-9939(09)10187-9

Article electronically published on December 31, 2009

\title{
WEAK CONTINUITY \\ OF THE GAUSS-CODAZZI-RICCI SYSTEM FOR ISOMETRIC EMBEDDING
}

\author{
GUI-QIANG CHEN, MARSHALL SLEMROD, AND DEHUA WANG
}

(Communicated by Walter Craig)

\begin{abstract}
We establish the weak continuity of the Gauss-Coddazi-Ricci system for isometric embedding with respect to the uniform $L^{p}$-bounded solution sequence for $p>2$, which implies that the weak limit of the isometric embeddings of the manifold in a fixed coordinate chart is an isometric immersion. More generally, we establish a compensated compactness framework for the Gauss-Codazzi-Ricci system in differential geometry. That is, given any sequence of approximate solutions to this system which is uniformly bounded in $L^{2}$ and has reasonable bounds on the errors made in the approximation (the errors are confined in a compact subset of $H_{\text {loc }}^{-1}$ ), the approximating sequence has a weakly convergent subsequence whose limit is a solution of the GaussCodazzi-Ricci system. Furthermore, a minimizing problem is proposed as a selection criterion. For these, no restriction on the Riemann curvature tensor is made.
\end{abstract}

\section{INTRODUCTION}

The Gauss-Codazzi-Ricci system is a fundamental system of nonlinear partial differential equations in differential geometry (cf. [4, 5, 12, 14, 15, 23, 25]). For example, the fundamental theorem of the surface theory indicates that the existence of a local or global solution of the Gauss-Codazzi-Ricci system can yield a local or global higher dimensional isometric embedding. Therefore, it is important to understand the behavior of this nonlinear system for solving isometric embedding problems and other important geometric problems. In general, the Gauss-CodazziRicci system has no type, neither purely hyperbolic nor purely elliptic.

Received by the editors August 14, 2009, and, in revised form, August 27, 2009.

2010 Mathematics Subject Classification. Primary 53C42, 53C21, 53C45, 35L65, 35M10, 35B35; Secondary 53C24, 57R40, 57R42, 58J32.

Key words and phrases. Weak continuity, Gauss-Codazzi-Ricci system, isometric embedding, weak convergence, approximate solutions, compensated compactness, Div-Curl lemma, minimization problem, selection criterion, Riemann curvature tensor.

(C)2009 American Mathematical Society Reverts to public domain 28 years from publication 
We are concerned with the weak continuity of the Gauss-Codazzi-Ricci system and the related compensated compactness framework for approximate solutions to this system. In Chen-Slemrod-Wang [8], we noted that the Gauss-Codazzi equations for isometric embedding of $\mathbb{M}^{2}$ into $\mathbb{R}^{3}$ fall naturally within the formulation of compensated compactness. In this paper, we first show that this is also true in the general case for the Gauss-Codazzi-Ricci system. One of our main observations here is that the Codazzi and Ricci equations naturally have the Div-Curl structure. Based on this observation, we establish the weak continuity of this system with respect to the uniform $L^{p}$-bounded solution sequence for $p>2$, which implies that the weak limit of the isometric embeddings of the manifold is an isometric immersion. This is reminiscent of the weak continuity of determinants which plays an essential role in the theory of polyconvexity by Ball [1] in nonlinear elasticity (also see Dacorogna 9], Evans [13, Morrey [19, and Müller [20]). More generally, we establish a stronger compensated compactness framework for the Gauss-CodazziRicci system. That is, given any sequence of approximate solutions to this system which is uniformly bounded in $L^{2}$ and has reasonable bounds on the errors made in the approximation (the errors are confined in a compact subset of $H_{\mathrm{loc}}^{-1}$ ), the approximating sequence has a weakly convergent subsequence whose limit is still a solution of the Gauss-Codazzi-Ricci system. For these estimates, no restriction on the Riemann curvature tensor is made.

A long-standing fundamental problem in differential geometry is the existence of local (and if possible global) embeddings of a $d$-dimensional Riemannian manifold $\mathbb{M}^{d}, d \geq 3$, into the Euclidean space $\mathbb{R}^{N}$ with optimal dimension $N$. As noted in Han-Hong [17, the first global existence of smooth $C^{\infty}$ embeddings was given by Nash [24], but the best result as of this time is the following theorem of Günther 16: Any smooth d-dimensional compact Riemannian manifold admits a smooth (i.e. $C^{\infty}$ ) isometric embedding in $\mathbb{R}^{N}$ for $N=\frac{1}{2} \max \{d(d+5), d(d+3)+10\}$. The proof of Günther is based on first doing a local existence proof for a fixed local coordinate system and then assembling the local results to obtain a global result (see 17 for a presentation). Needless to say, it is of considerable interest to know if Günther's dimension $N$ is optimal. In a similar vein, we could try to formulate a selection or "admissibility" criterion to choose one of the possibly infinite embeddings provided by Günther's theorem. Within the realm of surface theory and elastic manifolds, this has been recently considered in 11, 28 where the selection is done by minimizing an integral of the norm of the second fundamental form. Indeed, this seems to be a natural approach for selection in the general case and is even in the same spirit as Dafermos's entropy rate criterion [10. In Section 4, we propose a minimizing problem as a selection criterion. Specifically we show in the case where we have a sequence of embeddings defined by one fixed set of local coordinates $g_{i j} d x^{i} d x^{j}=d s^{2}, \mathbf{x} \in \Omega$, that by the compensated compactness framework any minimizing sequence has a subsequence in $L^{p}, p>2$, which converges weakly to a minimizer that satisfies the Gauss-Codazzi-Ricci system. Since any sequence of isometric embeddings of $\mathbb{M}^{d}$ into $\mathbb{R}^{N}$ (say given by Günther's theorem) must satisfy the equations exactly, this implies that the problem of minimizing the $L^{p}$-norms of the second fundamental form and the connection form on the normal bundle (sometimes called torsion coefficients [5]) does have a solution within the class of weak solutions of the Gauss-Codazzi-Ricci system, hence yielding an isometric immersion of $W^{2, p}$ class for $p>2$. 


\section{The Gauss-Codazzi-Ricci System for isometric EMbedding OF $\mathbb{M}^{d}$ INTO $\mathbb{R}^{N}$}

In this section, we use the following conventional notation:

$g_{i j}$ : given metric of the Riemannian manifold,

$\Gamma_{i j}^{k}$ : Christoffel symbols,

$R_{i j k l}:$ Riemann curvature tensor,

$h_{i j}^{a}$ : coefficients of the second fundamental form,

$\kappa_{l b}^{a}$ : coefficients of the connection form (torsion coefficients) on the normal bundle,

where the indices $a, b, c$ run from 1 to $N$ and $i, j, k, l, m, n$ run from 1 to $d \geq 3$.

For a given metric $g_{i j}$, the Christoffel symbols are

$$
\Gamma_{i j}^{k}=\frac{1}{2} g^{k l}\left(\partial_{j} g_{i l}+\partial_{i} g_{j l}-\partial_{l} g_{i j}\right)
$$

which depend on the first derivatives of $\left(g_{i j}\right)$, and the Riemann curvature tensor is

$$
R_{i j k l}=g_{l m}\left(\partial_{k} \Gamma_{i j}^{m}-\partial_{j} \Gamma_{i k}^{m}+\Gamma_{i j}^{n} \Gamma_{n k}^{m}-\Gamma_{i k}^{n} \Gamma_{n j}^{m}\right),
$$

which depends on $\left(g_{i j}\right)$ and its first and second derivatives, where $\left(g^{k l}\right)$ denotes the inverse of $\left(g_{i j}\right)$ and $\partial_{j}=\partial_{x_{j}}$. We denote $|g|=\operatorname{det}\left(g_{i j}\right)$.

2.1. The Gauss-Codazzi-Ricci system. As is well-known in Riemannian geometry, the isometric embedding problems for $d$-dimensional Riemannian manifolds into the Euclidean space $\mathbb{R}^{N}$ can be reduced to the solvability problems of the Gauss-Codazzi-Ricci system of nonlinear partial differential equations with the following form in local coordinates $g_{i j} d x^{i} d x^{j}=d s^{2}$ :

The Gauss equations:

$$
h_{j i}^{a} h_{k l}^{a}-h_{k i}^{a} h_{j l}^{a}=R_{i j k l}
$$

The Codazzi equations:

$$
\frac{\partial h_{l j}^{a}}{\partial x^{k}}-\frac{\partial h_{k j}^{a}}{\partial x^{l}}+\Gamma_{l j}^{m} h_{k m}^{a}-\Gamma_{k j}^{m} h_{l m}^{a}+\kappa_{k b}^{a} h_{l j}^{b}-\kappa_{l b}^{a} h_{k j}^{b}=0 ;
$$

The Ricci equations:

$$
\frac{\partial \kappa_{l b}^{a}}{\partial x^{k}}-\frac{\partial \kappa_{k b}^{a}}{\partial x^{l}}-g^{m n}\left(h_{m l}^{a} h_{k n}^{b}-h_{m k}^{a} h_{l n}^{b}\right)+\kappa_{k c}^{a} \kappa_{l b}^{c}-\kappa_{l c}^{a} \kappa_{k b}^{c}=0 .
$$

Notice that the coefficients of the second fundamental form are symmetric:

$$
h_{i j}^{a}=h_{j i}^{a},
$$

while the coefficients of the connection form on the normal bundle are antisymmetric:

$$
\kappa_{k b}^{a}=-\kappa_{k a}^{b} .
$$

In particular, the antisymmetry of $\kappa_{k b}^{a}$ implies

$$
\kappa_{k a}^{a}=-\kappa_{k a}^{a}
$$

and so

$$
\kappa_{k a}^{a}=0 .
$$

Thus, the ath column of the $d \times d$ matrix $\kappa^{a}$ is zero. 
When $d=3$, the Janet dimension $N=\frac{d(d+1)}{2}=6$ (cf. Janet [18]). Then

$$
\kappa^{1}=\left[\begin{array}{lll}
0 & \kappa_{12}^{1} & \kappa_{13}^{1} \\
0 & \kappa_{22}^{1} & \kappa_{23}^{1} \\
0 & \kappa_{32}^{1} & \kappa_{33}^{1}
\end{array}\right], \quad \kappa^{2}=\left[\begin{array}{ccc}
-\kappa_{12}^{1} & 0 & \kappa_{13}^{2} \\
-\kappa_{22}^{1} & 0 & \kappa_{23}^{2} \\
-\kappa_{32}^{1} & 0 & \kappa_{33}^{2}
\end{array}\right], \quad \kappa^{3}=\left[\begin{array}{ccc}
-\kappa_{13}^{1} & -\kappa_{13}^{2} & 0 \\
-\kappa_{23}^{1} & -\kappa_{23}^{2} & 0 \\
-\kappa_{33}^{1} & -\kappa_{33}^{2} & 0
\end{array}\right] .
$$

2.2. The Div-Curl structure of the Codazzi and Ricci equations. In this section we present one of our main observations on the features of the Codazzi and Ricci equations: the Div-Curl structure, which leads to the weak continuity of the system.

For $w=\left(w_{1}, w_{2}, \cdots, w_{d}\right)$,

$$
\operatorname{curl} w:=\left(\partial_{j} w_{i}-\partial_{i} w_{j}\right)_{1 \leq i, j \leq d}
$$

is a $d \times d$ matrix field.

From the Codazzi equations (2.2), for $k<l$, they possess the form

or

$$
\frac{\partial h_{l j}^{a}}{\partial x^{k}}-\frac{\partial h_{k j}^{a}}{\partial x^{l}}+\text { l.o.t. }=0
$$

$$
\operatorname{div}(\overbrace{l}^{\overbrace{0, \cdots, h_{l j}^{a}}^{k}, 0, \cdots,-h_{k j}^{a}}, 0, \cdots, 0)+\text { l.o.t. }=0
$$

and

$$
\operatorname{curl}\left(h_{1 j}^{a}, h_{2 j}^{a}, \cdots, h_{d j}^{a}\right)+\text { l.o.t. }=0,
$$

where l.o.t. represents the lower-order terms without involving derivatives in the equation.

Similarly, we observe that the identical form of the Ricci equations (2.3) can also be written as

$$
\operatorname{div}(\overbrace{\underbrace{0, \cdots, 0, \kappa_{l b}^{a}}_{l}, 0, \cdots,-\kappa_{k b}^{a}}^{k}, 0, \cdots, 0)+\text { l.o.t. }=0
$$

and

$$
\operatorname{curl}\left(\kappa_{1 b}^{a}, \kappa_{2 b}^{a}, \cdots, \kappa_{d b}^{a}\right)+\text { l.o.t. }=0 .
$$

Now replacing $a$ by $b$ and $j$ by $i$ in the Codazzi equations (2.6)-(2.7), we obtain

$$
\operatorname{div}(\overbrace{l}^{\overbrace{0, \cdots, h_{l i}^{b}}^{k}, 0, \cdots,-h_{k i}^{b}}, 0, \cdots, 0)+\text { l.o.t. }=0
$$

and

$$
\operatorname{curl}\left(h_{1 i}^{b}, h_{2 i}^{b}, \cdots, h_{d i}^{b}\right)+\text { l.o.t. }=0 .
$$

Similarly, replacing $a$ by $b$ and $b$ by $c$ in the Ricci equations (2.8)-(2.9), we have

$$
\operatorname{div}(\overbrace{l}^{\overbrace{0, \cdots, 0, \kappa_{l c}^{b}}^{k}, 0, \cdots,-\kappa_{k c}^{b}}, 0, \cdots, 0)+\text { l.o.t. }=0
$$


and

$$
\operatorname{curl}\left(\kappa_{1 c}^{b}, \kappa_{2 c}^{b}, \cdots, \kappa_{d c}^{b}\right)+\text { l.o.t. }=0 .
$$

One of our main observations is that the scalar products of the two vector fields in the rewritten forms (2.6)-(2.13) yield the nonlinear quantities in the lower-order terms in the Gauss-Codazzi-Ricci system (2.1)-(2.3): Forms (2.6) and (2.11) yield

$$
h_{l j}^{a} h_{k i}^{b}-h_{k j}^{a} h_{l i}^{b}
$$

forms (2.8) and (2.13) yield

$$
\kappa_{k b}^{a} \kappa_{l c}^{b}-\kappa_{l b}^{a} \kappa_{k c}^{b}
$$

and forms (2.9) and (2.10) yield

$$
\kappa_{k b}^{a} h_{l i}^{b}-\kappa_{l b}^{a} h_{k i}^{b}
$$

This observation is essential for us to establish the weak continuity of the GaussCodazzi-Ricci system in Section 3.

\section{WEAK CONTINUITY AND COMPENSATED COMPACTNESS FRAMEWORK}

In this section we establish the weak continuity of the Gauss-Codazzi-Ricci system and the related compensated compactness framework for approximate solutions to the system via the Div-Curl lemma (see Murat [21] and Tartar [26]).

The Div-Curl lemma is a basic result in the compensated compactness theory for the weak continuity of the scalar product of two vector fields (cf. [9, 13, 21, 22, 26. 27]) and is closely related to the Hodge decomposition.

Theorem 3.1 (Div-Curl lemma). Let $\Omega \subset \mathbb{R}^{d}, d \geq 2$, be open bounded. Let $p, q>1$ such that $\frac{1}{p}+\frac{1}{q}=1$. Assume that, for any $\varepsilon>0$, two fields $u^{\varepsilon} \in L^{p}\left(\Omega ; \mathbb{R}^{d}\right)$ and $v^{\varepsilon} \in L^{q}\left(\Omega ; \mathbb{R}^{d}\right)$ satisfy the following:

(i) $u^{\varepsilon} \rightarrow u$ weakly in $L^{p}\left(\Omega ; \mathbb{R}^{d}\right)$ as $\varepsilon \rightarrow 0$;

(ii) $v^{\varepsilon} \rightarrow v$ weakly in $L^{q}\left(\Omega ; \mathbb{R}^{d}\right)$ as $\varepsilon \rightarrow 0$;

(iii) $\operatorname{div} u^{\varepsilon}$ are confined in a compact subset of $W_{l o c}^{-1, p}(\Omega ; \mathbb{R})$;

(iv) $\operatorname{curl} v^{\varepsilon}$ are confined in a compact subset of $W_{l o c}^{-1, q}\left(\Omega ; \mathbb{R}^{d \times d}\right)$.

Then the scalar products of $u^{\varepsilon}$ and $v^{\varepsilon}$ are weakly continuous,

$$
u^{\varepsilon} \cdot v^{\varepsilon} \longrightarrow u \cdot v
$$

in the sense of distributions.

Based on our observation of the Div-Curl structure of the Codazzi and Ricci equations, we employ the Div-Curl lemma to formulate the following compensated compactness framework.

Let a sequence of vector fields $\left(h_{i j}^{a, \varepsilon}, \kappa_{l b}^{a, \varepsilon}\right)(\mathbf{x})$, defined on an open bounded subset $\Omega \subset \mathbb{R}^{d}$, satisfy the following Framework $(\mathrm{A})$ :

(A.1) $\left\|\left(h_{i j}^{a, \varepsilon}, \kappa_{l b}^{a, \varepsilon}\right)\right\|_{L^{2}(\Omega)} \leq C$ for some $C>0$ independent of $\varepsilon>0$.

(A.2) $\frac{\partial h_{l j}^{a, \varepsilon}}{\partial x^{k}}-\frac{\partial h_{k j}^{a, \varepsilon}}{\partial x^{l}}$ and $\frac{\partial \kappa_{l b}^{a, \varepsilon}}{\partial x^{k}}-\frac{\partial \kappa_{k b}^{a, \varepsilon}}{\partial x^{l}}$ are confined in a compact set in $H_{l o c}^{-1}(\Omega)$. 
(A.3) There exist $o_{j}^{\varepsilon}(1), j=1,2,3$, with $o_{j}^{\varepsilon}(1) \rightarrow 0$ in the sense of distributions as $\varepsilon \rightarrow 0$ such that

$$
\begin{aligned}
& \frac{\partial h_{l j}^{a, \varepsilon}}{\partial x^{k}}-\frac{\partial h_{k j}^{a, \varepsilon}}{\partial x^{l}}+\Gamma_{l j}^{m} h_{k m}^{a, \varepsilon}-\Gamma_{k j}^{m} h_{l m}^{a, \varepsilon}+\kappa_{k b}^{a, \varepsilon} h_{l j}^{b, \varepsilon}-\kappa_{l b}^{a, \varepsilon} h_{k j}^{b, \varepsilon}=o_{1}^{\varepsilon}(1), \\
& \frac{\partial \kappa_{l b}^{a, \varepsilon}}{\partial x^{k}}-\frac{\partial \kappa_{k b}^{a, \varepsilon}}{\partial x^{l}}-g^{m n}\left(h_{m l}^{a, \varepsilon} h_{k n}^{b, \varepsilon}-h_{m k}^{a, \varepsilon} h_{l n}^{b, \varepsilon}\right)+\kappa_{k c}^{a, \varepsilon} \kappa_{l b}^{c, \varepsilon}-\kappa_{l c}^{a, \varepsilon} \kappa_{k b}^{c, \varepsilon}=o_{2}^{\varepsilon}(1),
\end{aligned}
$$

and

$$
h_{j i}^{a, \varepsilon} h_{k l}^{a, \varepsilon}-h_{k i}^{a, \varepsilon} h_{j l}^{a, \varepsilon}=R_{i j k l}+o_{3}^{\varepsilon}(1) .
$$

Then we have

Theorem 3.2 (Compensated compactness framework). Let a sequence of vector fields $\left(h_{i j}^{a, \varepsilon}, \kappa_{l b}^{a, \varepsilon}\right)$ satisfy Framework (A). Then there exists a subsequence (still labeled) $\left(h_{i j}^{a, \varepsilon}, \kappa_{l b}^{a, \varepsilon}\right)$ that converges weakly in $L^{2}(\Omega)$ to $\left(h_{i j}^{a}, \kappa_{l b}^{a}\right)$ as $\varepsilon \rightarrow 0$ such that

(i) $\left\|\left(h_{i j}^{a}, \kappa_{l b}^{a}\right)\right\|_{L^{2}(\Omega)} \leq C$;

(ii) the quadratic terms in (2.1)-(2.3) are weakly continuous with respect to the subsequence $\left(h_{i j}^{a, \varepsilon}, \kappa_{l b}^{a, \varepsilon}\right)$ that converges to $\left(h_{i j}^{a}, \kappa_{l b}^{a}\right)$ weakly in $L^{2}(\Omega)$ as $\varepsilon \rightarrow 0$

(iii) the limit vector field $\left(h_{i j}^{a}, \kappa_{l b}^{a}\right)$ satisfies the Gauss-Codazzi-Ricci system (2.1) - (2.3)

That is, the limit vector field $\left(h_{i j}^{a}, \kappa_{l b}^{a}\right)$ is a weak solution to the Gauss-Codazzi-Ricci system (2.1) -(2.3).

Proof. By assumption (A.1), there exist a subsequence (still denoted) $\left(h_{i j}^{a, \varepsilon}, \kappa_{l b}^{a, \varepsilon}\right)$ and a vector field $\left(h_{i j}^{a}, \kappa_{l b}^{a}\right) \in L^{2}(\Omega)$ such that

$$
\left(h_{i j}^{a, \varepsilon}, \kappa_{l b}^{a, \varepsilon}\right) \rightarrow\left(h_{i j}^{a}, \kappa_{l b}^{a}\right) \quad \text { in } L^{2}(\Omega)
$$

and

$$
\left\|\left(h_{i j}^{a}, \kappa_{l b}^{a}\right)\right\|_{L^{2}(\Omega)} \leq C .
$$

By the Div-Curl structure, observed in Section 2.2, assumption (A.2) implies that

$$
\operatorname{div}(\overbrace{l}^{\overbrace{0, \cdots, h_{l j}^{a, \varepsilon}}^{0}, 0, \cdots,-h_{k j}^{a, \varepsilon}}, 0, \cdots, 0), \quad \operatorname{curl}\left(h_{1 j}^{a, \varepsilon}, h_{2 j}^{a, \varepsilon}, \cdots, h_{d j}^{a, \varepsilon}\right)
$$

and

$$
\operatorname{div}(\overbrace{l}^{\overbrace{0, \cdots, 0, \kappa_{l b}^{a, \varepsilon},}^{0, \cdots, \cdots,-\kappa_{k b}^{a, \varepsilon}}}, 0, \cdots, 0), \quad \operatorname{curl}\left(\kappa_{1 b}^{a, \varepsilon}, \kappa_{2 b}^{a, \varepsilon}, \cdots, \kappa_{d b}^{a, \varepsilon}\right)
$$

are confined in a compact set in $H_{l o c}^{-1}(\Omega)$.

By exchanging the indices, we also have that

$$
\operatorname{div}(\overbrace{l}^{\overbrace{0, \cdots, h_{l i}^{b, \varepsilon}}^{0,0}, \cdots,-h_{k i}^{b, \varepsilon}}, 0, \cdots, 0), \quad \operatorname{curl}\left(h_{1 i}^{b, \varepsilon}, h_{2 i}^{b, \varepsilon}, \cdots, h_{d i}^{b, \varepsilon}\right)
$$


and

$$
\operatorname{div}(\overbrace{l}^{\overbrace{0, \cdots, 0, \kappa_{l c}^{b, \varepsilon}}^{0, \cdots}, 0, \cdots,-\kappa_{k c}^{b, \varepsilon}}, 0, \cdots, 0), \quad \operatorname{curl}\left(\kappa_{1 c}^{b, \varepsilon}, \kappa_{2 c}^{b, \varepsilon}, \cdots, \kappa_{d c}^{b, \varepsilon}\right)
$$

are confined in a compact set in $H_{l o c}^{-1}(\Omega)$.

Using the Div-Curl lemma, Theorem 3.1, we conclude the weak continuity of the nonlinear quadratic quantities in the Gauss-Codazzi-Ricci system with respect to the sequence $\left(h_{i j}^{a, \varepsilon}, \kappa_{l b}^{a, \varepsilon}\right)$ :

$$
\begin{aligned}
& h_{l j}^{a, \varepsilon} h_{k i}^{b, \varepsilon}-h_{k j}^{a, \varepsilon} h_{l i}^{b, \varepsilon} \rightarrow h_{l j}^{a} h_{k i}^{b}-h_{k j}^{a} h_{l i}^{b}, \\
& \kappa_{k b}^{a, \varepsilon} \kappa_{l c}^{b, \varepsilon}-\kappa_{l b}^{a, \varepsilon} \kappa_{k c}^{b, \varepsilon} \rightarrow \kappa_{k b}^{a} \kappa_{l c}^{b}-\kappa_{l b}^{a} \kappa_{k c}^{b} \\
& \kappa_{k b}^{a, \varepsilon} h_{l i}^{b, \varepsilon}-\kappa_{l b}^{a, \varepsilon} h_{k i}^{b, \varepsilon} \rightarrow \kappa_{k b}^{a} h_{l i}^{b}-\kappa_{l b}^{a} h_{k i}^{b}
\end{aligned}
$$

in the sense of distributions as $\varepsilon \rightarrow 0$.

Combining (3.3)-(3.4) with (3.9)-(3.11), we conclude that the weak limit vector field $\left(h_{i j}^{a}, \kappa_{l b}^{a}\right)$ of the sequence $\left(h_{i j}^{a, \varepsilon}, \kappa_{l b}^{a, \varepsilon}\right)$ satisfies the Gauss-Codazzi-Ricci system (2.1) - (2.3) in the sense of distributions; that is, the limit vector field $\left(h_{i j}^{a}, \kappa_{l b}^{a}\right)$ is a weak solution of (2.1)- (2.3).

As a corollary, we conclude the weak continuity of the Gauss-Codazzi-Ricci system with respect to the uniform $L^{p}$-bounded solution sequence for $p>2$.

Theorem 3.3 (Weak continuity). Let $\left(h_{i j}^{a, \varepsilon}, \kappa_{l b}^{a, \varepsilon}\right)$ be a sequence of solutions to the Gauss-Codazzi-Ricci system (2.1)-(2.3), which is uniformly bounded in $L^{p}, p>2$. Then the weak limit vector field $\left(h_{i j}^{a}, \kappa_{l b}^{a}\right)$ of the sequence $\left(h_{i j}^{a, \varepsilon}, \kappa_{l b}^{a, \varepsilon}\right)$ in $L^{p}$ is still a solution to (2.1) -(2.3).

Proof. Since the solution sequence $\left(h_{i j}^{a, \varepsilon}, \kappa_{l b}^{a, \varepsilon}\right)$ is uniformly bounded in $L^{p}, p>2$,

$$
\left\|\left(h_{i j}^{a, \varepsilon}, \kappa_{l b}^{a, \varepsilon}\right)\right\|_{L^{p}(\Omega)} \leq C,
$$

for some $C>0$ independent of $\varepsilon$, there exist a subsequence (still denoted) $\left(h_{i j}^{a, \varepsilon}, \kappa_{l b}^{a, \varepsilon}\right)$ and a vector field $\left(h_{i j}^{a}, \kappa_{l b}^{a}\right) \in L^{p}(\Omega)$ such that

$$
\left(h_{i j}^{a, \varepsilon}, \kappa_{l b}^{a, \varepsilon}\right) \rightarrow\left(h_{i j}^{a}, \kappa_{l b}^{a}\right) \quad \text { in } L^{p}(\Omega)
$$

and

$$
\left\|\left(h_{i j}^{a}, \kappa_{l b}^{a}\right)\right\|_{L^{p}(\Omega)} \leq C .
$$

Then we conclude from (3.12) that all the lower-order terms for the solution sequence $\left(h_{i j}^{a, \varepsilon}, \kappa_{l b}^{a, \varepsilon}\right)$ in the Gauss-Codazzi-Ricci system (2.1)-(2.3) are uniformly bounded in $L^{p / 2}, p>2$. This implies that

$$
\frac{\partial h_{l j}^{a}}{\partial x^{k}}-\frac{\partial h_{k j}^{a}}{\partial x^{l}}, \frac{\partial \kappa_{l b}^{a}}{\partial x^{k}}-\frac{\partial \kappa_{k b}^{a}}{\partial x^{l}} \text { are confined in a compact set in } H_{l o c}^{-1}(\Omega) .
$$

Since the domain $\Omega \subset \mathbb{R}^{d}$ is bounded, the uniform bound in (3.12) implies the uniform bound of $\left(h_{i j}^{a, \varepsilon}, \kappa_{l b}^{a, \varepsilon}\right)$ in $L^{2}(\Omega)$. By the compensated compactness framework (Theorem 3.2), we conclude that the limit vector field is a weak solution of (2.1) - (2.3), which implies the weak continuity of the system. 
Remark 3.1. The weak continuity of the Gauss-Codazzi-Ricci system implies that, for $p>2$, the weak limit of a sequence of isometric embeddings of the $d$-dimensional manifold $\mathbb{M}^{d}$ into $\mathbb{R}^{N}$ as surfaces with corresponding uniform $L^{p}$-bounded sequence $\left(h_{i j}^{a, \varepsilon}, \kappa_{l b}^{a, \varepsilon}\right)$ is an isometric immersion as a surface in $\mathbb{R}^{N}$. The requirement that $p>2$ is to ensure the $H^{-1}$-compactness in (3.13) to deal with the nonhomogeneous terms.

\section{Minimization PRoblem}

In this section, as an example, we show that the solution sequence $\left(h_{i j}^{a, \varepsilon}, \kappa_{l b}^{a, \varepsilon}\right)$ for the weak continuity in Theorem 3.3 can be obtained from a selection criterion. In particular, we wish to select an "energy" minimizer among all embeddings with local coordinates $g_{i j} d x^{i} d x^{j}=d s^{2}, x \in \Omega$. Non-uniqueness and uniqueness (rigidity) of embeddings were discussed by Berger-Bryant-Griffiths [2, 3].

Theorem 4.1. There exists a minimizer $\left(h_{i j}^{a}, \kappa_{l b}^{a}\right)$ for the minimization problem

$$
\min _{S}\|(h, \kappa)\|_{L^{p}(\Omega)}^{p}:=\min _{S} \int_{\Omega} \sqrt{|g|}\left(\left(h_{i j} h_{i j}\right)^{\frac{p}{2}}+\left(\kappa_{l b} \kappa_{l b}\right)^{\frac{p}{2}}\right) d x, \quad p>2,
$$

where $S$ is the set of weak solutions to the Gauss-Codazzi-Ricci system (2.1)-(2.3).

Proof. Clearly, $S$ is non-empty by the local version of Günther's theorem in [16] (also see Section 1.2 in [17] and the statement in Section 1 above). A minimizing sequence provides the desired $L^{p}$-norm for the weak continuity theorem (Theorem 3.3). Since the $L^{p}$-norm, which is weakly lower semicontinuous, is convex, any minimizing sequence has a subsequence in $L^{p}(\Omega)$ that converges weakly to a minimizer which satisfies the Gauss-Codazzi-Ricci system (2.1)-(2.3).

Notice that any sequence of isometric embeddings of $\mathbb{M}^{d}$ into $\mathbb{R}^{N}$ with a fixed local coordinate system $g_{i j} d x^{i} d x^{j}=d s^{2}, x \in \Omega$, as surfaces (say, given by Günther's theorem) must satisfy the Gauss-Codazzi-Ricci equations (2.1)-(2.3). This implies that the problem of minimizing the $L^{p}$-norms of the second fundamental form and the connection form on the normal bundle does have a solution within the class of weak solutions of the Gauss-Codazzi-Ricci system (2.1)-(2.3), hence yielding an isometric immersion of $W^{2, p}$ class for $p>2$ for $\mathbb{M}^{d}$ into $\mathbb{R}^{N}$ as a surface for $x \in \Omega$.

\section{ACKNOWLEDGMENTS}

The authors would like to thank Jeff Viaclovsky and the referee for their valuable remarks, which helped improve our earlier presentation.

The first author's research was supported in part by the National Science Foundation under Grants DMS-0807551, DMS-0720925, and DMS-0505473; the Natural Science Foundation of China under Grant NSFC-10728101; and the Royal SocietyWolfson Research Merit Award (UK).

The second author's research was supported in part by the National Science Foundation under Grant DMS-0647554.

The third author's research was supported in part by the National Science Foundation under Grants DMS-0604362 and DMS-0906160, and by the Office of Naval Research under Grant N00014-07-1-0668.

This paper was written as part of the International Research Program on Nonlinear Partial Differential Equations at the Centre for Advanced Study at the Norwegian Academy of Science and Letters in Oslo during the academic year 2008-09. 
It was finalized when the authors participated in the SQuaRE on "Isometric Embedding of Higher Dimensional Riemannian Manifolds", which was held at the American Institute of Mathematics, Palo Alto, California, March 16-20, 2009.

\section{REFERENCES}

[1] J. M. Ball, Convexity conditions and existence theorems in nonlinear elasticity, Arch. Rational Mech. Anal. 63 (1976/77), 337-403. MR0475169 (57:14788)

[2] E. Berger, R. Bryant, and P. Griffiths, Some isometric embedding and rigidity results for Riemannian manifolds, Proc. Nat. Acad. Sci. U.S.A. 78 (1981), 4657-4660. MR627257 (82h:53074)

[3] E. Berger, R. Bryant, and P. Griffiths, The Gauss equations and rigidity of isometric embeddings, Duke Math. J. 50 (1983), 803-892. MR714831 (85k:53056)

[4] R. L. Bryant, P. A. Griffiths, and D. Yang, Characteristics and existence of isometric embeddings, Duke Math. J. 50 (1983), 893-994. MR726313 (85d:53027)

[5] Yu. D. Burago and S. Z. Shefel, The geometry of surfaces in Euclidean spaces, Geometry III, 1-85, Encyclopaedia Math. Sci., 48, Burago and Zalggaller (eds.), Springer-Verlag: Berlin, 1992. MR1306734

[6] E. Cartan, Sur la possibilité de plonger un espace Riemannian donné dans un espace Euclidien, Ann. Soc. Pol. Math. 6 (1927), 1-7.

[7] B.-Y. Chen, Classification of locally symmetric spaces which admit a totally umbilical hypersurface, Soochow J. Math. 6 (1980), 39-48. MR626317 (82j:53096)

[8] G.-Q. Chen, M. Slemrod, and D. Wang, Isometric immersions and compensated compactness, Commun. Math. Phys., in press.

[9] B. Dacorogna, Weak Continuity and Weak Lower Semicontinuity of Nonlinear Functionals, Springer-Verlag: Berlin, 1982. MR658130 (84f:49020)

[10] C. M. Dafermos, Hyperbolic Conservation Laws in Continuum Physics, second edition, Springer-Verlag: Berlin, 2005. MR 2169977 (2006d:35159)

[11] B. A. DiDonna, T. A. Witten, S. C. Venkataramani, and E. M. Kramer, Singularities, structures, and scaling in deformed m-dimensional elastic manifolds. Phys. Rev. E (3) 65 (2002), 016603, 1-25. MR1877621(2002k:82091)

[12] L. P. Eisenhart, Riemannian Geometry, eighth printing, Princeton University Press: Princeton, NJ, 1997. MR1487892(98h:53001)

[13] L. C. Evans, Weak Convergence Methods for Nonlinear Partial Differential Equations, CBMS-RCSM, 74, Amer. Math. Soc.: Providence, RI, 1990. MR1034481 (91a:35009)

[14] H. F. Goenner, On the interdependency of the Gauss-Codazzi-Ricci equations of local isometric embedding, General Relativity and Gravitation 8 (1977), 139-145. MR0467606 (57:7462)

[15] R. Greene, Isometric embeddings of Riemannian and pseudo-Riemannian manifolds, Memoirs Amer. Math. Soc., 97, Amer. Math. Soc.: Providence, RI, 1970. MR0262980 (41:7585)

[16] M. Günther, On the perturbation problem associated to isometric embeddings of Riemannian manifolds, Ann. Global Anal. Geom. 7 (1989), 69-77. MR.1029846 (91a:58023)

[17] Q. Han and J.-X. Hong, Isometric Embedding of Riemannian Manifolds in Euclidean Spaces, Mathematical Surveys and Monographs, 130, Amer. Math. Soc.: Providence, RI, 2006. MR2261749(2008e:53055)

[18] M. Janet, Sur la possibilité de plonger un espace Riemannian donné dans un espace Euclidien, Ann. Soc. Pol. Math. 5 (1926), 38-43.

[19] C. B. Morrey, Multiple Integrals in the Calculus of Variations, Springer: Berlin, New York, 1966. MR0202511 (34:2380)

[20] S. Müller, A surprising higher integrability property of mappings with positive determinant, Bull. Amer. Math. Soc. (N.S.) 21 (1989), 245-248. MR999618 (90c:49022)

[21] F. Murat, Compacité par compensation, Ann. Scuola Norm. Sup. Pisa Cl. Sci. (4) 5 (1978), 489-507. MR506997 (80h:46043a)

[22] F. Murat, Compacité par compensation. II, in: Proceedings of the International Meeting on Recent Methods in Nonlinear Analysis (Rome, 1978), pp. 245-256, Pitagora: Bologna, 1979. MR.533170 (80h:46043b)

[23] G. Nakamura and Y. Maeda, Local isometric embedding problem of Riemannian 3-manifold into $\mathbb{R}^{6}$, Proc. Japan Acad. Ser. A: Math. Sci. 62 (1986), 257-259. MR.868813 (88b:53026) 
[24] J. Nash, The imbedding problem for Riemannian manifolds, Ann. of Math. (2) 63 (1956), 20-63. MR.0075639 (17:782b)

[25] M. Spivak, A Comprehensive Introduction to Differential Geometry, Publish or Perish, Inc.: Boston, MA, Vols. I-II, 1970; Vols. III-V, 1975. MR0532830 (82g:53003a), MR0532831 (82g:53003b) MR0532832(82g:53003c), MR0532833(82g:53003d), MR0532834 (82g:53003e)

[26] L. Tartar, Compensated compactness and applications to partial differential equations, in: Nonlinear Analysis and Mechanics: Heriot-Watt Symposium, Vol. IV, pp. 136-212, Res. Notes in Math., 39, Pitman: Boston, MA; London, 1979. MR.584398 (81m:35014)

[27] L. Tartar, The compensated compactness method applied to systems of conservation laws, in: Systems of Nonlinear Partial Differential Equations (Oxford, 1982), 263-285, NATO Adv. Sci. Inst. Ser. C Math. Phys. Sci., 111, Reidel: Dordrecht, 1983. MR.725524 (85e:35079)

[28] T. A. Witten, Stress focusing in elastic sheets, Reviews of Modern Physics 79 (2007), 643-675. MR2326936 (2008e:74052)

School of Mathematical Sciences, Fudan University, Shanghai 200433, People's Republic of China - And - Department of Mathematics, Northwestern University, Evanston, Illinois 60208 - And - Mathematical Institute, University of Oxford, OxFORD, OX1 3LB, UNiTED KingDOM

E-mail address: gqchen@math.northwestern.edu

Department of Mathematics, University of Wisconsin, Madison, Wisconsin 53706 and - Department of Mathematics, University of Pittsburgh, Pittsburgh, Pennsylvania 15260 - and - Department of Mathematical Sciences, Korean Advanced Institute for Science and Technology, Daejeon, Republic of Korea

E-mail address: slemrod@math.wisc.edu

Department of Mathematics, University of Pittsburgh, Pittsburgh, Pennsylvania 15260

E-mail address: dwang@math.pitt.edu 